\title{
Evaluation of morphometric characteristics and watershed prioritization of Bhadar basin of Saurashtra region, Gujarat
}

\begin{abstract}
J. H. Kelaiya
Department of Soil and Water Conservation Engineering, College of Agricultural Engineering and Technology, Junagadh Agricultural University, Junagadh (Gujarat), India H. D. Rank

Department of Soil and Water Conservation Engineering, College of Agricultural Engineering and Technology, Junagadh Agricultural University, Junagadh (Gujarat), India D. K. Dwivedi*

Department of Natural Resource Management, College of Forestry, Navsari Agricultural University, Navsari (Gujarat), India

*Corresponding author. E-mail:dhavaldwivedi42@gmail.com

Abstract

Morphometric analysis is one of the important aspects of quantitative geomorphology which is primarily used to study the geometrical aspects of the landforms. The study was undertaken with the objectives of evaluating morphometric characteristic and prioritizing the watersheds of Bhadar basin based on its morphometric characteristics. Linear, relief and aerial aspects were calculated for watershed characterization. The watersheds were ranked on the basis of high values of linear parameters and low values of shape parameters. A total of 16 watersheds 5G1B1, 5G1B2, 5G1B3, 5G1B4, 5G1B5, 5G1B6, 5G1B7, 5G1B8, 5G1B9, 5G1B10, 5G1B11, 5G1B12, 5G1B13, 5G1B14, 5G1B15 and 5G1B16 were identified in the Bhadar basin and morphometric characteristic of each watershed was determined. Highest priority indicated the greater degree of erosion in the particular watershed and it therefore priority should be given in applying soil conservation measures. It was concluded that the watershed 5G1B15 should be given highest priority because of higher erosion problems over other watersheds of Bhadar basin while 5G1B4 should be given the least priority.
\end{abstract}

Keywords: Geographic Information System, Morphometric characteristics, Prioritization, Watershed

\section{INTRODUCTION}

The natural structure of any drainage basin and can be expressed in a quantitative way through measurement of aerial, linear and relief aspect (Horton, 1945). Morphometric analysis represents the analysis of the configuration of the shape and dimension of landforms of earth (Agarwal, 1998). The quantitative expression of drainage basin morphometry was first presented by Horton (1945) and after that, Strahler (1952) developed a stream order system. The quantitative analysis of morphometric parameters is has tremendous applications in evaluating river basin, prioritization of watersheds and management of natural resources (Malik et al., 2011). The evaluation of morphometric characteristics is possible from analysing drainage parameters such as basin area, perimeter, stream orders, length of drainage channels, drainage density, stream frequency, bifurcation ratio, elongation and circulation ratio, texture ratio, basin relief, slope ratio, ruggedness number and length

\section{Article Info}

DOI: 10.31018/jans.v11i2.2032

Received: March 5, 2019

Revised: April 4, 2019

Accepted: April 12, 2019

\section{How to Cite}

Kelaiya, J.H. et al. (2019). Evaluation of morphometric characteristics and watershed prioritization of Bhadar basin of Saurashtra region, Gujarat. Journal of Applied and Natural Science, 11(2): 273- 280 https://doi.org/10.31018/ jans.v11i2.2032 of over land flow (Kumar et al., 2000; Nag and Chakraborthy, 2003). Morphometric analysis in a drainage basin is important for hydrological investigation and management of drainage basin (Rekha et al. 2011). By field observation alone, it is difficult to examine all drainage networks due to their extent throughout basin and therefore advanced tools of Geographic Information System (GIS) is useful for extracting and evaluating the characteristic of the basin in terms of its hydrological response (Ali et al., 2017). The morphometric characteristics gives an insight to the problems associated with watersheds such as runoff and erosion and therefore, these morphometric characteristics are useful in prioritizing the watersheds which needs appropriate soil and water conservation measures.

Varade et al. (2018) analysed the morphometric characteristics of Dhaneri Watershed, Gambhar River Basin, Himachal Pradesh, India. The study area of Dhaneri watershed has been divided into 
seven sub-watersheds (I to VII) and investigated with respect to various morphometric aspects Various morphometric aspects of Dhaneri watershed showed good correlation, which indicated that the soil characteristics of the study area were governed by surface erosion phenomena. Relative weightage system involving hierarchal rankings to various morphometric aspects was used to evolve compound values showing low, medium and high land priority zones in the study area. It was concluded that the sub-watersheds ' $\mathrm{V}$ ' and 'VI' needed formulation of proper development plans for harnessing their natural resources on urgent basis. Farhan et al. (2018) carried out prioritization of 76 fourth-order sub-watersheds using morphometric analysis of linear and shape parameters in a semi-arid drainage basin in Southern Jordan using GIS, morphometric analysis and multivariate statistics. Ranks were designated to each subwatershed on the basis of the calculated compound parameter. The total score for each subbasin was calculated based on the threat of erosion. Appropriate soil and water conservation measures should be planned based on the results to achieve agricultural sustainability in the study region. The study was undertaken with the objectives of evaluating the morphometric characteristics of the watersheds of Bhadar basin and prioritizing the watersheds based on their morphometric characteristics.

Study area: Saurashtra is the western peninsular region of Gujarat, which covers an area of about 58,743 square $\mathrm{km}$ lying over Arabian Sea Coast, with the coastline of about $925 \mathrm{~km}$ length. In Saurashtra, Bhadar is one of the major rivers that drains about $1 / 7$ th of the Saurashtra area. The Bhadar basin is situated between $21^{\circ} 25^{\prime}$ to $22^{\circ}$ $10^{\prime}$ North latitude and $69^{\circ} 45^{\prime}$ to $71^{\circ} 20^{\prime}$ ' East longitude. The location of the study area is given in Fig. 1.

\section{MATERIALS AND METHODS}

This section includes the methods used for morphometric analysis of watersheds and prioritization. The ArcGis software was used for calculation of the morphometric characteristics. The SRTM (Shuttle Radar Topography Mission) 30-m image of Saurashtra region with study area was used for the GIS interpretation (http:// earthexplorer.usgs.gov/).

Morphometric analysis of watershed: The drainage map of basin was opened into the ArcMap environment. The stream ordering was done in ArcMap manually using Editor tool. Each stream segment was edited and stream order was given in attribute table simultaneously. The tools like Flip and Merge were used to change the direction of flow and to join the stream segment, respectively. Simultaneously the cartosat image of Gujarat state with study area was used to view the flow direction of each stream segment. The drainage map was then clipped into 16 watersheds using clipping tool for further geomorphological analysis. Linear aspects, Aerial aspects and relief aspects of watershed were calculated using the following equations given in Table 1.

Watershed prioritization: These linear parameters such as Bifurcation ratio $\left(R_{b}\right)$, Stream Frequency $\left(F_{s}\right)$, Length of overland flow $\left(L_{g}\right)$, Texture Ratio $(T)$ Drainage Density $\left(D_{d}\right)$, and relief parameters like relief, relative relief and relief ratio bear a direct relationship with erodibility. Therefore, higher values of the linear parameters indicate higher erodibility. For watershed prioritization, rank 1 was assigned to the highest value of linear parameters, rank 2 was assigned to the second highest linear parameter value and so on, and the last rank was given to the least value of linear parameters. Shape parameters such as Elongation Ratio (Re), Form Factor (Rf), Circulatory Ratio $\left(R_{c}\right)$ and Compactness Coefficient $\left(C_{c}\right)$ do not have a direct relationship with erodibility; they are inversely related (Ratnam et al., 2005). Therefore, if the value of shape parameters is low then it is an indication of high erodibility. Rank 1 was given to lowest shape parameter values, rank 2 was given to the next lower value of shape parameters and so on. A compound value $\left(C_{p}\right)$ was obtained once the ranks considering the linear and shape parameters were added for each sub-watershed. Least priority was given to the watershed with the highest value of compound parameter and viceversa.

\section{RESULTS AND DISCUSSION}

The various geomorphologic parameters like linear, shape and relief parameters of the Bhadar river basin area were determined and summarized in Table 2 to Table 7 .

The Bhadar river basin was divided into 16 water-

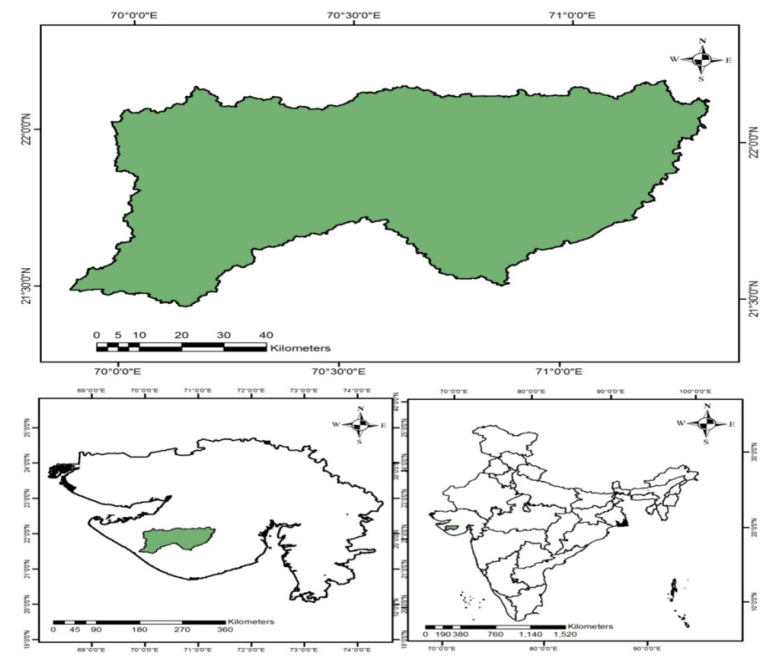

Fig. 1. Location map of Bhadar basin of Saurashtra region in Gujarat. 


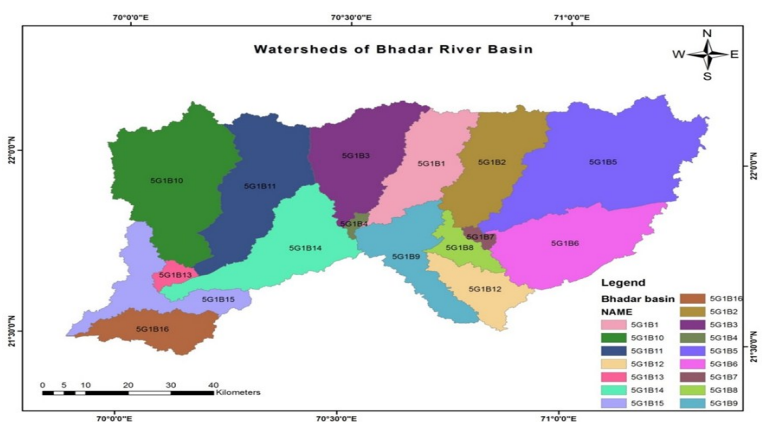

Fig. 2. Watershed map of Bhadar river basin (ArcGIS).

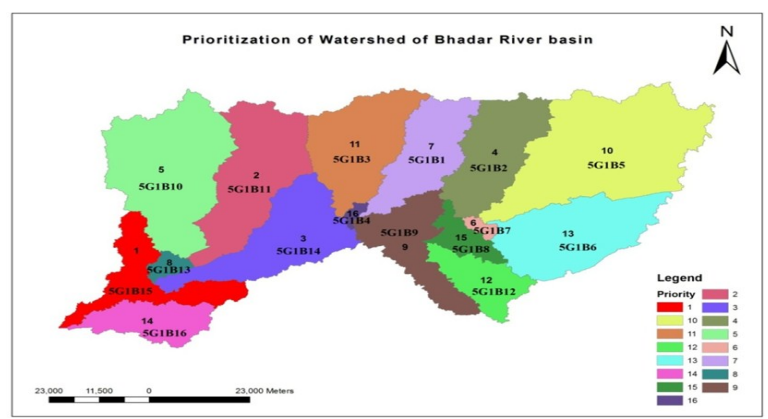

Fig. 3. Prioritization of watershed of Bhadar river sheds with codes shown in Fig. 2. The codes of the sub-watersheds are 5G1B1,5G1B2, 5G1B3, 5G1B4, 5G1B5, 5G1B6, 5G1B7, 5G1B8, 5G1B9, 5G1B10, 5G1B11, 5G1B12, 5G1B13, 5G1B14, $5 \mathrm{G} 1 \mathrm{~B} 15$ and 5G1B16.

The prioritization map of watershed of Bhadar river basin is shown in Fig. 3.

The Bhadar river basin is of $7^{\text {th }}$ order basin. The watersheds $5 \mathrm{G} 1 \mathrm{~B} 15$, consisted of $1^{\text {st }}$ to $7^{\text {th }}$ stream orders. The higher amount stream order indicated lesser permeability and infiltration in these watersheds. Drainage patterns of stream network from the basin have been observed as mainly dendritic type which indicated the texture homogeneity. The bifurcation ratio $(\mathrm{Rb})$ ranged from 1.50 to 12 which meant that the geologic structures did not distort the drainage pattern..All watersheds showed drainage density more than $2 \mathrm{~km} / \mathrm{km}^{2}$ which indicated the presence of impermeable sub-surface, bare vegetation and high relief. The stream frequency for all 16 watersheds of the study area exhibited correlation which was positive with the drainage density which indicated that with the increase of drainage density stream population increased. The watersheds were found to be elongated in nature

Table 1. Morphometric parameters used for watershed characterization of Bhadar.

\begin{tabular}{|c|c|c|c|}
\hline $\begin{array}{l}\text { Sr. } \\
\text { No. }\end{array}$ & $\begin{array}{l}\text { Morphometric parame- } \\
\text { ters }\end{array}$ & Formula & Reference \\
\hline 1 & Stream Order & Hierarchical & Strahler (1964) \\
\hline 2 & Stream Length, $\mathrm{Km}\left(\mathrm{L}_{\mathrm{u}}\right)$ & Length of stream & Horton (1945) \\
\hline 3 & $\begin{array}{l}\text { Mean stream } \\
\text { length }\left(L_{s m}\right)\end{array}$ & $\begin{array}{l}\mathrm{Lsm}=\mathrm{L}_{\mathrm{u}} / \mathrm{N}_{\mathrm{u}} \\
\text { Where, } \mathrm{L}_{u}=\text { total stream length of order } u \\
\text { Total number of stream segments of order ' } u \text { ' }\end{array}$ & Strahler (1964) \\
\hline 4 & $\begin{array}{l}\text { Stream Length } \\
\text { ratio }(R L)\end{array}$ & $\begin{array}{l}\mathrm{RL}=\mathrm{L}_{\mathrm{u}} /\left(\mathrm{L}_{\mathrm{u}-1}\right) \\
\text { Where, Lu= Total stream length of order ' } u \\
\mathrm{u}-1=\text { 'otal no of stream segments of its Next lower order }\end{array}$ & Horton (1945) \\
\hline 5 & $\begin{array}{l}\text { Bifurcation ratio } \\
\left(R_{b}\right)\end{array}$ & $\begin{array}{l}N_{b}=N_{u} /\left(N_{u+1}\right) \\
\text { Where, } N_{u}=\text { Number stream segments of order'u' } N_{u+1}=\text { Number of } \\
\text { stream segments of next higher order }\end{array}$ & Schumn (1956) \\
\hline 6 & $\begin{array}{l}\text { Mean bifurcation } \\
\text { ratio }\left(R_{h m}\right)\end{array}$ & Average of bifurcation ration of all orders & Strahler (1957) \\
\hline 7 & $\begin{array}{l}\text { Length of main } \\
\text { channel }\left(L_{m}\right) \mathrm{Km}\end{array}$ & $\begin{array}{l}\text { Length along longest water course form the outflow point of des- } \\
\text { ignated sub-basin to the upper limit of catchment boundary }\end{array}$ & Horton (1945) \\
\hline 8 & $\begin{array}{l}\text { Drainage Density } \\
\left(D_{d}\right)\end{array}$ & $\begin{array}{l}D_{d}=L_{u} / A \\
\text { Where, Lu=Total stream length of all orders, } \mathrm{km} \\
\qquad A=\text { Area of the Basin, } \mathrm{km}^{2}\end{array}$ & Horton (1932) \\
\hline 9 & $\begin{array}{l}\text { Length of } \\
\text { overland flow } \\
\left(L_{g}\right)\end{array}$ & $\begin{array}{l}\mathrm{Lg}=1 / \mathrm{D}_{\mathrm{d}-2} 2 \\
\text { Where, } \mathrm{D}_{\mathrm{d}}=\text { Drainage Density }\end{array}$ & Horton (1945) \\
\hline 10 & $\begin{array}{l}\text { Basin length (Lb) } \\
\mathrm{Km}\end{array}$ & Distance between outlet and farthest point on basin boundary & Horton (1945) \\
\hline 11 & Basin perimeter $(\mathrm{P}) \mathrm{Km}$ & Length of watershed divide which surround the Basin & Horton (1945) \\
\hline 12 & $\begin{array}{l}\text { Fineness ratio } \\
\left(R_{\mathrm{fn}}\right)\end{array}$ & $\begin{array}{l}\text { Rfn }=L_{b} / P \\
\text { Where, } L_{b}=\text { Basin length, } k m, P=\text { Basin perimeter, } \mathrm{km}\end{array}$ & Melton (1957) \\
\hline 13 & Basin/drainage area $(A)$ & Area enclosed within the boundary of watershed divide & Horton (1932) \\
\hline 14 & $\begin{array}{l}\text { Constant of channel } \\
\text { maintenance }(\mathrm{C})\end{array}$ & $\begin{array}{l}C=1 / D \\
\text { Where, } D=\text { Drainage Density, } \mathrm{km} / \mathrm{km}^{2} \\
\mathrm{FS}=\mathrm{N}_{4} / \mathrm{A}\end{array}$ & Horton (1932) \\
\hline 15 & Stream frequency $\left(F_{s}\right)$ & $\begin{array}{l}\text { Where, } \mathrm{N}_{\mathrm{u}}=\text { Total number of stream segments of all order, } \mathrm{A}= \\
\text { Area of the Basin, } \mathrm{km}^{2}\end{array}$ & Horton (1932) \\
\hline 16 & $\begin{array}{l}\text { Circulatory ratio } \\
\left(R_{c}\right)\end{array}$ & $\begin{array}{l}\mathrm{R} C=2 \mathrm{R} \times \mathrm{A} / \mathrm{P}^{2} \\
\text { Where, } \mathrm{RC}=\text { Circularity Ratio, } \mathrm{A}=\text { Area of the basin, } \mathrm{km}^{2} \\
\mathrm{Re}=2 \mathrm{R} / \mathrm{L}_{\mathrm{b}}\end{array}$ & Miller (1953) \\
\hline 17 & $\begin{array}{l}\text { Elongation ratio } \\
\left(\mathrm{R}_{\mathrm{e}}\right)\end{array}$ & $\begin{array}{l}\text { Where, } A \stackrel{=}{=} \text { Area of the basin, } \mathrm{km}^{2} \\
R=\text { radius of circle whose area equal to basin area, } \\
L_{b}=\text { Basin length }\end{array}$ & Schumm (1956) \\
\hline 18 & Form Factor( $\left.\mathrm{R}_{\mathrm{f}}\right)$ & $\begin{array}{l}\mathrm{Rf}=\mathrm{A} / \mathrm{L}_{\mathrm{b}}=\text { Area of the basin, } \mathrm{km}^{2}, L_{b}=\text { Basin length } \\
\text { Where, } A=\text {. }\end{array}$ & Horton (1932) \\
\hline 19 & Total relief $(\mathrm{H})$ & $\begin{array}{l}\mathrm{H}=\text { is the maximum vertical distance between the lowest (outlet) } \\
\text { and the highest (divide) points in the watershed. }\end{array}$ & Schumm (1956) \\
\hline 20 & Relief ratio $\left(R_{h}\right)$ & $\begin{array}{l}\mathrm{Rh}=\mathrm{H} / \mathrm{L}_{b} \\
\text { Where, } \mathrm{H}=\text { basin total relief, } \mathrm{L}_{b}=\text { basin Length }\end{array}$ & Schumm (1956) \\
\hline 21 & $\begin{array}{l}\text { Relative relief } \\
\left(R_{p}\right)\end{array}$ & $\begin{array}{l}\mathrm{R}_{\mathrm{p}}=\mathrm{H} / \mathrm{P} \\
\text { Where } \mathrm{H}=\text { total relief, } \mathrm{P}=\text { Perimeter }\end{array}$ & Melton (1957) \\
\hline
\end{tabular}


Table 2. Linear aspects of Bhadar river basin.

\begin{tabular}{|c|c|c|c|c|c|c|c|}
\hline \multicolumn{8}{|c|}{ Stream orders, numbers and length } \\
\hline $\begin{array}{l}\text { Stream } \\
\text { order }\end{array}$ & No of streams & & $\begin{array}{l}\text { Total length of } \\
\mathrm{km}\end{array}$ & streams, & $\begin{array}{l}\text { Mean } \\
\text { length, k }\end{array}$ & $\begin{array}{l}\text { stream } \\
\mathrm{rm}\end{array}$ & $\begin{array}{l}\text { Length of overland } \\
\text { flow km }\end{array}$ \\
\hline 1 & 3652 & & 10564.38 & & 2.89 & & 0.20 \\
\hline 2 & 1963 & & 4118.11 & & 2.10 & & \\
\hline 3 & 995 & & 1495.70 & & 1.50 & & \\
\hline 4 & 122 & & 482.49 & & 3.95 & & \\
\hline 5 & 18 & & 456.27 & & 25.35 & & \\
\hline 6 & 12 & & 1416.56 & & 118.05 & & \\
\hline 7 & 1 & & 17.21 & & 17.21 & & \\
\hline \multicolumn{8}{|c|}{ Bifurcation ratio $\left(\mathrm{N}_{\mathrm{u}} / \mathrm{N}_{\mathrm{u}+1}\right)$} \\
\hline $1^{\text {st }} / 2^{\text {nd }}$ & $2^{\text {nd }} / 3^{\text {rd }}$ & $3^{\mathrm{rd}} / 4^{\mathrm{th}}$ & $4^{\text {th }} / 5^{\text {th }}$ & \multicolumn{2}{|c|}{$5^{\text {th }} / 6^{\text {th }}$} & $6^{\text {th }} / 7^{\text {th }}$ & Mean \\
\hline 1.86 & 1.97 & 8.16 & 6.78 & 1.5 & & 12 & 5.38 \\
\hline \multicolumn{8}{|c|}{ Stream length ratio $\left(\mathrm{L}_{\mathrm{u}+1} / \mathrm{L}_{\mathrm{u}}\right)$} \\
\hline $2^{\text {nd }} / 1^{\mathrm{st}}$ & $3^{\mathrm{rd}} / 2^{\text {nd }}$ & $4^{\text {th }} / 3^{\text {rd }}$ & $5^{\mathrm{th}} / 4^{\mathrm{th}}$ & \multicolumn{2}{|c|}{$6^{\text {th }} / 5^{\text {th }}$} & $7^{\text {th }} / 6^{\text {th }}$ & Mean \\
\hline 0.73 & 0.72 & 2.63 & 6.41 & \multicolumn{2}{|c|}{4.66} & 0.15 & 2.55 \\
\hline
\end{tabular}

Table 3. Aerial aspects of Bhadar river basin.

\begin{tabular}{llllllll}
\hline \multicolumn{7}{c}{ Aerial aspects of basin } \\
\hline $\begin{array}{l}\text { Drainage } \\
\text { density } \\
\left(\mathbf{k m} / \mathbf{k m}^{2}\right)\end{array}$ & $\begin{array}{l}\text { Stream fre- } \\
\text { quency } \\
\mathbf{k m})\end{array}$ & $\begin{array}{l}\text { Circularity } \\
\text { ratio }\end{array}$ & $\begin{array}{l}\text { Compactness } \\
\text { coefficient }\end{array}$ & $\begin{array}{l}\text { Form } \\
\text { factor }\end{array}$ & $\begin{array}{l}\text { Elongation } \\
\text { ratio }\end{array}$ & $\begin{array}{l}\text { Drainage } \\
\text { texture } \\
(\mathbf{1} / \mathbf{k m})\end{array}$ \\
\hline 2.53 & 0.92 & 0.011198 & 9.44 & 0.01136 & 0.12030 & 2.36 \\
\hline
\end{tabular}

Table 4. Relief aspects of Bhadar river basin.

\begin{tabular}{|c|c|c|c|c|}
\hline \multicolumn{5}{|c|}{ Relief parameters of basin } \\
\hline Relief, km & Relief ratio & Relative & relief $\quad$ Channel slope $\mathrm{km} / \mathrm{km}$ & slope, $\mathrm{km} / \mathrm{km}$ \\
\hline 0.303 & 0.000377 & 0.01056 & 0.24 & 0.77 \\
\hline
\end{tabular}

Table 5. Linear aspects of watersheds of Bhadar river basin.

\begin{tabular}{|c|c|c|c|c|c|}
\hline \multicolumn{6}{|c|}{ A. Stream orders, numbers and length } \\
\hline watershed & $\begin{array}{l}\text { Stream } \\
\text { order }\end{array}$ & No of streams & $\begin{array}{l}\text { Total length of } \\
\text { streams, } \mathrm{km}\end{array}$ & Mean stream length, $\mathbf{k m}$ & $\begin{array}{l}\text { Length of } \\
\text { overland } \\
\text { flow, } \mathrm{km}\end{array}$ \\
\hline \multirow{6}{*}{ 5G1B1 } & 1 & 228 & 686.18 & 3.01 & \multirow{6}{*}{0.18} \\
\hline & 2 & 131 & 281.85 & 2.15 & \\
\hline & 3 & 47 & 72.59 & 1.54 & \\
\hline & 4 & 5 & 34.41 & 6.88 & \\
\hline & 5 & 1 & 36.55 & 36.55 & \\
\hline & 6 & 1 & 145.09 & 145.09 & \\
\hline \multirow{5}{*}{ 5G1B2 } & 1 & 280 & 810.66 & 2.89 & \multirow{5}{*}{0.20} \\
\hline & 2 & 158 & 329.38 & 2.08 & \\
\hline & 3 & 75 & 117.68 & 1.57 & \\
\hline & 4 & 42 & 32.95 & 0.78 & \\
\hline & 5 & 1 & 32.04 & 32.04 & \\
\hline \multirow{5}{*}{ 5G1B3 } & 1 & 324 & 950.73 & 2.93 & \multirow{5}{*}{0.20} \\
\hline & 2 & 165 & 343.50 & 2.08 & \\
\hline & 3 & 91 & 135.76 & 1.49 & \\
\hline & 4 & 6 & 35.16 & 5.86 & \\
\hline & 5 & 1 & 39.99 & 39.99 & \\
\hline \multirow{6}{*}{ 5G1B4 } & 1 & 10 & 33.94 & 3.39 & \multirow{6}{*}{0.05} \\
\hline & 2 & 5 & 11.56 & 2.31 & \\
\hline & 3 & 0 & 0 & 0 & \\
\hline & 4 & 0 & 0 & 0 & \\
\hline & 5 & 0 & 0 & 0 & \\
\hline & 6 & 1 & 3.91 & 3.91 & \\
\hline \multirow{6}{*}{ 5G1B5 } & 1 & 533 & 1476.33 & 2.76 & \multirow{6}{*}{0.23} \\
\hline & 2 & 296 & 600.95 & 2.03 & \\
\hline & 3 & 142 & 220.54 & 1.55 & \\
\hline & 4 & 13 & 103.03 & 7.92 & \\
\hline & 5 & 3 & 34.78 & 11.59 & \\
\hline & 6 & 1 & 145.10 & 145.09 & \\
\hline
\end{tabular}


Kelaiya, J.H. et al. / J. Appl. \& Nat. Sci. 11(2): 273- 280 (2019)

\begin{tabular}{|c|c|c|c|c|c|c|}
\hline & 1 & & 327 & 934.56 & 2.85 & \\
\hline & 2 & & 176 & 373.23 & 2.12 & \\
\hline \multirow[t]{4}{*}{ 5G1B6 } & 3 & & 111 & 165.63 & 1.49 & 0.21 \\
\hline & 4 & & 4 & 38.78 & 9.69 & \\
\hline & 5 & & 1 & 24.36 & 24.36 & \\
\hline & 1 & & 21 & 75.67 & 3.60 & \\
\hline \multirow{4}{*}{ 5G1B7 } & 2 & & 5 & 14.91 & 2.98 & 005 \\
\hline & 3 & & 2 & 2.97 & 1.48 & 0.05 \\
\hline & 6 & & 1 & 8.10 & 8.10 & \\
\hline & 1 & & 77 & 259.88 & 3.37 & \\
\hline \multirow{5}{*}{ 5G1B8 } & 2 & & 37 & 71.04 & 1.92 & 012 \\
\hline & 3 & & 31 & 49.37 & 1.59 & 0.12 \\
\hline & 6 & & 1 & 12.12 & 12.12 & \\
\hline & 1 & & 217 & 631.44 & 2.91 & \\
\hline & 2 & & 110 & 219.54 & 1.99 & \\
\hline \multirow{7}{*}{ 5G1B9 } & 3 & & 60 & 90.20 & 1.50 & 0.18 \\
\hline & 4 & & 29 & 21.10 & 0.72 & 0.10 \\
\hline & 5 & & 1 & 0.015 & 0.015 & \\
\hline & 6 & & 1 & 20.99 & 20.99 & \\
\hline & 1 & & 445 & 1235.20 & 2.77 & \\
\hline & 2 & & 247 & 481.78 & 1.95 & \\
\hline & 3 & & 140 & 215.29 & 1.53 & \\
\hline \multirow[t]{6}{*}{ 5G1B10 } & 4 & & 6 & 72.97 & 12.16 & 0.23 \\
\hline & 5 & & 2 & 20.48 & 10.24 & \\
\hline & 6 & & 1 & 36.89 & 36.89 & \\
\hline & 7 & & 0 & 0 & 0 & \\
\hline & 1 & & 354 & 995.30 & 2.81 & \\
\hline & 2 & & 172 & 372.09 & 2.16 & \\
\hline \multirow[t]{5}{*}{ 5G1B11 } & 3 & & 135 & 187.73 & 1.39 & 0.22 \\
\hline & 4 & & 4 & 25.41 & 6.35 & \\
\hline & 5 & & 1 & 50.87 & 50.87 & \\
\hline & 1 & & 137 & 427.57 & 3.12 & \\
\hline & 2 & & 68 & 157.73 & 2.31 & \\
\hline \multirow[t]{5}{*}{ 5G1B12 } & 3 & & 20 & 27.72 & 1.38 & 0.16 \\
\hline & 4 & & 2 & 27.70 & 13.85 & \\
\hline & 5 & & 1 & 11.07 & 11.07 & \\
\hline & 1 & & 36 & 97.16 & 2.69 & \\
\hline & 2 & & 19 & 48.78 & 2.56 & \\
\hline \multirow{6}{*}{ 5G1B13 } & 3 & & 3 & 3.21 & 1.07 & 0.12 \\
\hline & 4 & & 0 & 0 & 0 & \\
\hline & 5 & & 1 & 50.87 & 50.87 & \\
\hline & 6 & & 1 & 36.89 & 36.89 & \\
\hline & 1 & & 305 & 874.83 & 2.86 & \\
\hline & 2 & & 170 & 366.36 & 2.15 & \\
\hline \multirow{6}{*}{ 5G1B14 } & 3 & & 63 & 95.02 & 1.50 & \\
\hline & 4 & & 6 & 40.30 & 6.71 & 0.19 \\
\hline & 5 & & 1 & 39.99 & 39.99 & \\
\hline & 6 & & 1 & 145.09 & 145.10 & \\
\hline & 1 & & 208 & 635.69 & 3.06 & \\
\hline & 2 & & 120 & 245.28 & 2.04 & \\
\hline \multirow{6}{*}{ 5G1B15 } & 3 & & 46 & 62.52 & 1.36 & 016 \\
\hline & 4 & & 2 & 36.43 & 18.21 & 0.16 \\
\hline & 6 & & 2 & 0.037 & 0.018 & \\
\hline & 7 & & 1 & 17.20 & 17.20 & \\
\hline & 1 & & 150 & 439.19 & 2.92 & \\
\hline & 2 & & 84 & 200.05 & 2.38 & \\
\hline 5G1B16 & 3 & & 29 & 49.41 & 1.70 & 0.20 \\
\hline & 4 & & 3 & 14.20 & 4.73 & \\
\hline & 5 & & 1 & 11.18 & 11.18 & \\
\hline B. Bifurcati & atio & & & & & \\
\hline Watershed & $1^{\text {st }} / 2^{\text {nd }}$ & $2^{\text {nd }} / 3^{\text {rd }}$ & $3^{\text {rd }} / 4^{\text {th }}$ & $4^{\text {th }} / 5^{\text {th }}$ & $5^{\text {th }} / 6^{\text {th }}$ & $6^{\text {th }} / 7^{\text {th }}$ \\
\hline 5G1B1 & 1.74 & 2.78 & 9.4 & 5 & 1 & 3.98 \\
\hline 5G1B2 & 1.77 & 2.10 & 1.78 & 42 & - & 11.91 \\
\hline 5G1B3 & 1.96 & 1.81 & 15.16 & 6 & - & 6.23 \\
\hline 5G1B4 & 2 & - & - & - & 1 & 1.5 \\
\hline 5G1B5 & 1.80 & 2.08 & 10.92 & 4.33 & 3 & 4.43 \\
\hline 5G1B6 & 1.85 & 1.58 & 27.75 & 4 & - & 8.80 \\
\hline 5G1B7 & 4.2 & 2.5 & - & - & 1 & 2.56 \\
\hline 5G1B8 & 2.08 & 1.19 & - & - & 1 & 1.42 \\
\hline 5G1B9 & 1.97 & 1.83 & 2.06 & 29 & 1 & 7.17 \\
\hline & & & & & & Contd.. \\
\hline
\end{tabular}


Kelaiya, J.H. et al. / J. Appl. \& Nat. Sci. 11(2): 273- 280 (2019)

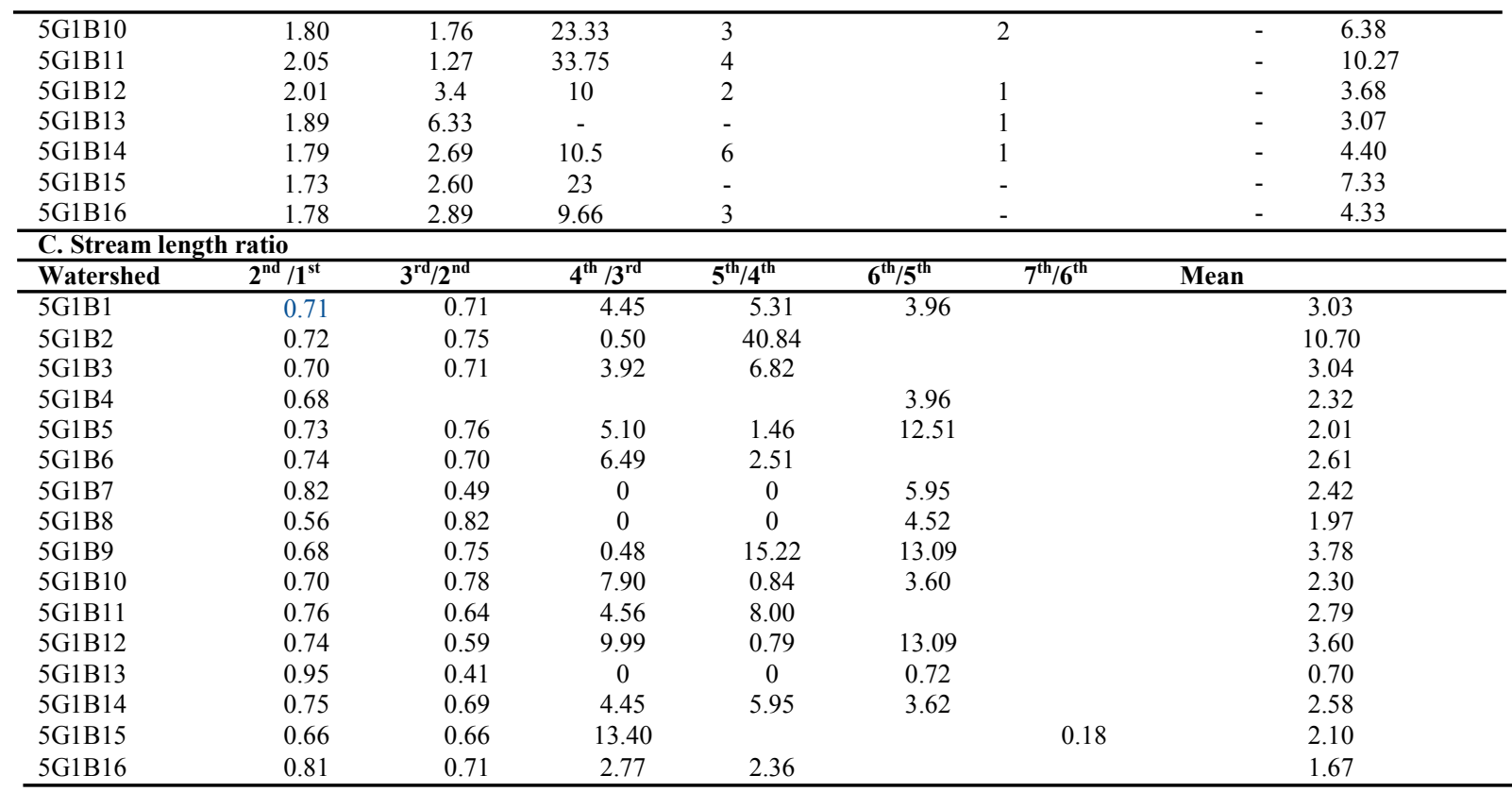

Table 6. Aerial aspects of watersheds of Bhadar river basin.

\begin{tabular}{|c|c|c|c|c|c|c|c|}
\hline Watershed & $\begin{array}{l}\text { Drainage } \\
\text { density } \\
\left(\mathbf{k m} / \mathrm{km}^{2}\right)\end{array}$ & $\begin{array}{lr}\begin{array}{l}\text { Stream } \\
\text { quensy }\end{array} & \text { fre- } \\
\left.\mathrm{km}^{2}\right) & \\
\end{array}$ & $\begin{array}{l}\text { Elonga- } \\
\text { tion ratio }\end{array}$ & $\begin{array}{l}\text { Circularity } \\
\text { ratio }\end{array}$ & $\begin{array}{l}\text { Form } \\
\text { factor }\end{array}$ & $\begin{array}{l}\text { Compactness } \\
\text { coefficient }\end{array}$ & $\begin{array}{l}\text { Drainage } \\
\text { texture } \\
(1 / \mathrm{km})\end{array}$ \\
\hline 5G1B1 & 2.74 & 0.90 & 0.40 & 0.14 & 0.13 & 2.64 & 2.05 \\
\hline 5G1B2 & 2.51 & 1.05 & 0.42 & 0.16 & 0.14 & 2.46 & 2.76 \\
\hline 5G1B3 & 2.49 & 0.97 & 0.47 & 0.19 & 0.18 & 2.25 & 2.98 \\
\hline 5G1B4 & 9.96 & 0.74 & 0.56 & 0.20 & 0.25 & 2.21 & 0.45 \\
\hline 5G1B5 & 2.21 & 0.81 & 0.46 & 0.16 & 0.17 & 2.50 & 3.18 \\
\hline 5G1B6 & 2.30 & 0.92 & 0.50 & 0.20 & 0.20 & 2.23 & 3.03 \\
\hline 5G1B7 & 8.41 & 0.95 & 0.38 & 0.18 & 0.12 & 2.34 & 0.64 \\
\hline 5G1B8 & 4.06 & 1.07 & 0.47 & 0.12 & 0.18 & 2.82 & 1.25 \\
\hline 5G1B9 & 2.71 & 1.01 & 0.45 & 0.11 & 0.16 & 3.04 & 1.91 \\
\hline 5G1B10 & 2.17 & 0.88 & 0.47 & 0.20 & 0.17 & 2.24 & 3.42 \\
\hline 5G1B11 & 2.28 & 0.93 & 0.40 & 0.16 & 0.12 & 2.47 & 2.84 \\
\hline 5G1B12 & 3.04 & 0.87 & 0.46 & 0.15 & 0.17 & 2.60 & 1.54 \\
\hline 5G1B13 & 4.25 & 1.07 & 0.45 & 0.22 & 0.16 & 2.10 & 1.07 \\
\hline 5G1B14 & 2.57 & 0.90 & 0.41 & 0.14 & 0.13 & 2.61 & 2.39 \\
\hline 5G1B15 & 3.04 & 0.97 & 0.38 & 0.06 & 0.11 & 3.83 & 1.41 \\
\hline 5G1B16 & 2.54 & 0.95 & 0.42 & 0.14 & 0.14 & 2.61 & 1.71 \\
\hline
\end{tabular}

Table 7. Relief aspects of watersheds of Bhadar river basin.

\begin{tabular}{|c|c|c|c|c|c|}
\hline Watershed & Relief km & $\begin{array}{l}\text { Relative } \quad \text { relief } \\
\text { km/km }\end{array}$ & Relief ratio & $\begin{array}{l}\text { Channel slope } \\
\text { km/km }\end{array}$ & $\begin{array}{l}\text { Ground slope, } \\
\text { km/km }\end{array}$ \\
\hline 5G1B1 & 0.185 & 0.0922 & 0.00307 & 0.00195 & 0.00307 \\
\hline 5G1B2 & 0.186 & 0.0926 & 0.00306 & 0.00195 & 0.00306 \\
\hline 5G1B3 & 0.144 & 0.0732 & 0.00246 & 0.00157 & 0.00246 \\
\hline 5G1B4 & 0.051 & 0.135 & 0.00532 & 0.00339 & 0.00532 \\
\hline 5G1B5 & 0.225 & 0.0725 & 0.00266 & 0.00170 & 0.00266 \\
\hline $5 \mathrm{G} 1 \mathrm{~B} 6$ & 0.144 & 0.0705 & 0.00249 & 0.00158 & 0.00249 \\
\hline 5G1B7 & 0.056 & 0.120 & 0.00344 & 0.00219 & 0.00344 \\
\hline 5G1B8 & 0.091 & 0.0775 & 0.00328 & 0.00209 & 0.00328 \\
\hline 5G1B9 & 0.139 & 0.0633 & 0.00277 & 0.00176 & 0.00277 \\
\hline 5G1B10 & 0.261 & 0.1063 & 0.00355 & 0.00226 & 0.00355 \\
\hline 5G1B11 & 0.246 & 0.1049 & 0.00325 & 0.00207 & 0.00325 \\
\hline $5 \mathrm{G} 1 \mathrm{~B} 12$ & 0.141 & 0.0949 & 0.00359 & 0.00229 & 0.00359 \\
\hline $5 \mathrm{G} 1 \mathrm{~B} 13$ & 0.065 & 0.1166 & 0.00346 & 0.00220 & 0.00346 \\
\hline $5 \mathrm{G} 1 \mathrm{~B} 14$ & 0.283 & 0.124 & 0.00415 & 0.00264 & 0.00415 \\
\hline 5G1B15 & 0.267 & 0.0999 & 0.00460 & 0.00293 & 0.00460 \\
\hline 5 G1B16 & 0.047 & 0.0302 & 0.00104 & 0.00066 & 0.00104 \\
\hline
\end{tabular}


i.e. they assumed a pear shaped characteristics indicating high degree of integration. The elongated watershed with low value of form factor indicated that the basin had a flatter peak flow for longer duration. The low circularity ratio was observed in all watersheds indicating that they were more or less elongated in shape, which also indicated low discharge of runoff and highly permeability of the subsoil. Coarse texture was found in watershed 5G1B1, 5G1B2, 5G1B3, 5G1B5, 5G1B6, 5G1B10, 5G1B11 and 5G1B14 which mean that they had less runoff potential. The watersheds 5G1B4, 5G1B7, 5G1B8, 5G1B13 and 5G1B16 were of low relief region and remaining were of moderate relief region. It was noticed that the lower values in case of most watershed indicated the existence of basement rocks. In another study Wandre et al. (2015) morphometric characteristics were used for prioritization of Shetrunji river basin. Such study could prove very useful for watershed planners to implement the appropriate soil and water conservation measures to the prioritized watershed.

\section{Conclusion}

The individual values of compound parameters for the watersheds 5G1B1, 5G1B2, 5G1B3, 5G1B4, 5G1B5, 5G1B6, 5G1B7, 5G1B8, 5G1B9, 5G1B10, 5G1B11，5G1B12，5G1B13，5G1B14，5G1B15 and 5G1B16 were found as $8.25,7.00,9.33$, $10.83,9.25,9.75,8.16,10.33,9.00,7.66,6.25$, $9.41,8.41,6.5,5.66$ and 10.16 respectively. Priority of watershed was allotted on the basis of compound parameter value. That means that the level of soil erosion increased with decrease in values of compound parameters or the priority increased with decrease in their values (Mishra et al, 2010). The ascending order of priority of watersheds was 5G1B7, 5G1B4, 5G1B11, 5G1B16, 5G1B10，5G1B13，5G1B6，5G1B15，5G1B9, 5G1B5, 5G1B2, 5G1B12, 5G1B8, 5G1B3, 5G1B1 and $5 \mathrm{G} 1 \mathrm{~B} 14$ respectively. Therefore the watershed 5G1B15 should be treated first while 5G1B4 at last. Highest priority indicated the greater degree of erosion in the particular watershed and it therefore priority should be given in applying soil conservation measures.

\section{REFERENCES}

1. Agarwal, C. S. (1998). Study of drainage pattern through aerial data in Naugarh area of Varanasi district, UP. Journal of the Indian Society of Remote Sensing, 26(4): 169-175. https://doi.org/10.1007/ BF02990795

2. Ali, K., Bajracharya, R. M., Sitaula, B. K., Raut, N., and Koirala, H. L. (2017). Morphometric Analysis of Gilgit River Basin in Mountainous Region of GilgitBaltistan Province, Northern Pakistan. Journal of Geoscience and Environment Protection, 5 (07) : 70. DOI: $10.4236 / g e p .2017 .57008$

3. Anonymous. (1879). The United States Geological Survey (USGS, formerly simply Geological Survey) is a scientific agency of the United States government. Virginia, United States. Retrieved from http: earthexplorer.usgs.gov/

4. Farhan, Y., Anbar, A., Al-Shaikh, N., Almohammad H., Alshawamreh, S., and Barghouthi, M. (2018). Prioritization of Sub-Watersheds in a Large Semi-Arid Drainage Basin (Southern Jordan) Using Morphometric Analysis, GIS, and Multivariate Statistics. Agricultural Sciences, 9(04), 437. DOI: 10.4236/ as. 2018.94031

5. Horton, R. (1932). Drainage Basin Characteristics, Transactions. American Geophysical Union, 13: 350361. https://doi.org/10.1029/TR013i001p00350

6. Horton, R. (1945). Erosional Development of Streams and Their Drainage Basins, Hydrophysical Approach to Quantitative Morphology. Geological Society of America Bulletin, 56, 275-370. https:// doi.org/10.1130/0016-7606(1945)56[275:EDOSAT] 2.0.CO;2

7. Kumar, R., Kumar, S., Lohani, A. K., Nema, R. K., and Singh, R. D. (2000). Evaluation of geomorphological characteristics of a catchment using GIS. G/s India, 9(3): 13-17.

8. Malik, M., Bhat, M. and Kuchay, N. (2011) Watershed based drainage morphometric analysis of Lidder catchment in Kashmir valley using geographical information system. Recent Res Sci Technol 3(4):118126.

9. Melton, M. A. (1957). An analysis of the relations among elements of climate, surface properties, and geomorphology (No. CU-TR-11). COLUMBIA UNIV NEW YORK.

10.Miller, V.C. (1953) A Quantitative Geomorphologic Study of Drainage Basin Characteristics in the Clinch Mountain Area, Virginia and Tennessee, Columbia University Department of Geology, ONR Geography Branch, New York. Project NR 389042, Tech Rept 3.

11.Nag, S. K., and Chakraborty, S. (2003). Influence of rock types and structures in the development of drainage network in hard rock area. Journal of the Indian Society of Remote Sensing, 31(1): 25-35. https://doi.org/10.1007/BF03030749

12.Ratnam, K. N., Srivastava, Y. K., Rao, V. V., Amminedu, E. and Murthy, K. S. R. (2005). Check dam positioning by prioritization of micro-watersheds using SYI model and morphometric analysis-remote sensing and GIS perspective. Journal of the Indian society of remote sensing, 33(1):25. https://doi.org/10.1007/ BF02989988

13.Rekha, B. V., George, A. V., and Rita, M. (2011). Morphometric analysis and micro-watershed prioritization of Peruvanthanam sub-watershed, the Manimala River Basin, Kerala, South India. Environmental Research, Engineering and Management, 57(3): 614.

14.Schumn S.A. (1956). Evolution of drainage systems and slopes in badlands at Perth Amboy, New Jersey, Geological Society of America Bulletin.67(5):597-646. https://doi.org/10.1130/0016-7606(1956)67 [597:EODSAS]2.0.CO;2

15.Strahler, A. N. (1957). Quantitative analysis of watershed geomorphology. Transactions American Geophysical Union, 38: 913-920. https://doi.org/10.1029/ TR038i006p00913

16.Strahler, A.N. (1952) Hypsometric Analysis of Erosional Topography. Bulletin of the Geological Society of America, 63: 1117-1142. https:// 
Kelaiya, J.H. et al. / J. Appl. \& Nat. Sci. 11(2): 273- 280 (2019)

doi.org/10.1130/0016-7606(1952)63[1117:HAAOET]

2.0.CO;2

17.Strahler, A.N. (1964). Quantitative geomorphology of drainage basins and channel networks. In: V.T. Chow (ed), Handbook of Applied Hydrology. McGraw Hill Book Company, New York, section 4-II.773.

18.Varade, A. M., Kale, H. S., Pachpor, S., and Thakur,
N. G. (2018). Morphometric Analysis of Dhaneri Watershed, Gambhar River Basin, Himachal Pradesh, India. Journal of Geosciences, 3(1): 59-65.

19. Wandre, S.S, Rank, H. D., and Shinde, V. B. (2015). Prioritization of watersheds of shetrunji river basin based on morphometric analysis using remote sensing and GIS. Multilogic in science, 4(12): 24-30. 